\title{
ARTICLE OPEN Separate tuning of nematicity and spin fluctuations to unravel the origin of superconductivity in FeSe
}

\author{
Seung-Ho Baek $\mathbb{D}^{1 *}$, Jong Mok $\mathrm{Ok}^{2,3}$, Jun Sung Kim${ }^{2,3}$, Saicharan Aswartham $\mathbb{D}^{4}$, Igor Morozov ${ }^{4,5}$, Dmitriy Chareev ${ }^{6,7,8}$, \\ Takahiro Urata $^{9,10}$, Katsumi Tanigaki ${ }^{9}$, Yoichi Tanabe ${ }^{9,11}$, Bernd Büchner ${ }^{4,12}$ and Dmitri V. Efremov ${ }^{4}$
}

The interplay of orbital and spin degrees of freedom is the fundamental characteristic in numerous condensed matter phenomena, including high-temperature superconductivity, quantum spin liquids, and topological semimetals. In iron-based superconductors (FeSCs), this causes superconductivity to emerge in the vicinity of two other instabilities: nematic and magnetic. Unveiling the mutual relationship among nematic order, spin fluctuations, and superconductivity has been a major challenge for research in FeSCs, but it is still controversial. Here, by carrying out ${ }^{77}$ Se nuclear magnetic resonance (NMR) measurements on FeSe single crystals, doped by cobalt and sulfur that serve as control parameters, we demonstrate that the superconducting transition temperature $T_{\mathrm{c}}$ increases in proportion to the strength of spin fluctuations, while it is independent of the nematic transition temperature $T_{\text {nem. }}$. Our observation therefore directly implies that superconductivity in FeSe is essentially driven by spin fluctuations in the intermediate coupling regime, while nematic fluctuations have a marginal impact on $T_{c}$.

npj Quantum Materials (2020)5:8; https://doi.org/10.1038/s41535-020-0211-y

\section{INTRODUCTION}

In correlated Fermi fluids, nematicity refers to the state in which rotational symmetry is spontaneously broken, while time-reversal invariance is preserved, and consequently, the symmetry of the crystal changes from tetragonal to orthorhombic. ${ }^{1}$ An important aspect in iron-based superconductors (FeSCs) is the propensity for nematic ordering, which is usually followed by a spin-densitywave (SDW) transition, near a superconducting (SC) dome. ${ }^{2-4}$ Regardless of the origin of nematicity that is still under debate, ${ }^{5,6}$ this raises the fundamental issue of whether superconductivity in FeSCs is closely related to nematicity ${ }^{7,8}$ or magnetism ${ }^{9,10}$ or both. ${ }^{11}$ To address this issue, it is much desirable to separate nematic order from the magnetic one. In this respect, FeSe has been a key platform for studying the origin of nematicity and its role in superconductivity, ${ }^{12}$ as it exhibits nematic and SC orders at well-separated temperatures, $T_{\text {nem }} \sim 90 \mathrm{~K}$ and $T_{\mathrm{c}} \sim 9 \mathrm{~K}$, respectively, without involving magnetic order. Numerous recent studies in FeSe show that nematicity causes the strongly anisotropic SC gap symmetry, ${ }^{13-16}$ and further discuss that nematic fluctuations might play an important role for the superconducting pairing mechanism. ${ }^{17,18}$ On the other hand, the leading role of spin fluctuations (SFs) in the SC mechanism of FeSe, as in other FeSCs whose parent materials magnetical orders, has been also proposed in the literature. ${ }^{19-22}$ In this spin fluctuation-mediated pairing scenario, the subsequent question arises whether weak or strong coupling approach is appropriate to establish theory of superconductivity in FeSCs. It is quite interesting to note that recent NMR studies of FeSe under high pressure reveal the persistence of local nematicity at temperatures far above $T_{\text {nem, }}$ which suggests a correlation between local nematicity and magnetism. ${ }^{23,24}$ Another interesting observation by NMR is the unusual suppression of $\left(T_{1} T\right)^{-1}$ at optimal pressure, ${ }^{25}$ suggesting that the interplay of SFs and superconductivity may undergo a critical change with high pressure.

As a system undergoes a nematic transition $\left(C_{4} \rightarrow C_{2}\right)$, two nematic domains are naturally formed below $T_{\text {nem, }}$, still preserving the $C_{4}$ symmetry on average. Accordingly, it is usually required to detwin nematic domains, for example, by an external strain to study nematicity. As a local probe in real space, on the other hand, NMR is uniquely capable of observing the two nematic domains at the same time. Indeed, it has been established that the splitting of the NMR line in FeSCs at an external field $H$ applied along the crystallographic $a$ axis represents the nematic order parameter and its onset temperature corresponds to the nematic transition temperature $T_{\text {nem }}$ (refs., ${ }^{26-28}$ see Fig. 1c). In order to investigate whether and how nematicity is related to superconductivity, we measured the ${ }^{77} \mathrm{Se}$ line splitting for $H_{\| a}$ in $\mathrm{FeSe}_{1-y} \mathrm{~S}_{y}$ and $\mathrm{Fe}_{1-x} \mathrm{Co}_{x} \mathrm{Se}$ single crystals. In general, it is considered that substituting isovalent $\mathrm{S}$ for $\mathrm{Se}$ is equivalent to the application of (negative) chemical pressure, and Co substituted for Fe supplies an additional electron and also plays as a paramagnetic impurity. Therefore, a systematic NMR study on the two different doped systems may enable a full understanding of the relationship between nematicity, magnetism, and superconductivity.

\section{RESULTS AND DISCUSSION}

Figure 1 shows how the temperature dependence of the ${ }^{77} \mathrm{Se}$ NMR spectrum in FeSe is modified as a function of $x$ in $\mathrm{Fe}_{1-x} \mathrm{Co}_{x} \mathrm{Se}$ (Fig. 1a, b) and as a function of $y$ in $\mathrm{FeSe}_{1-y} \mathrm{~S}_{y}$ (Fig. $1 d$, e). For FeSe ${ }_{1-y} S_{y}$, we find that the onset temperature of the line splitting or $T_{\text {nem }}$ is gradually suppressed, consistent with previous studies. ${ }^{1,21}$ For $\mathrm{Fe}_{1-x} \mathrm{Co}_{x} \mathrm{Se}$, however, $T_{\text {nem }}$ hardly

\footnotetext{
${ }^{1}$ Department of Physics, Changwon National University, Changwon 51139, Korea. ${ }^{2}$ Department of Physics, Pohang University of Science and Technology, Pohang 790-784, Korea. ${ }^{3}$ Center for Artificial Low Dimensional Electronic Systems, Institute of Basic Science, Pohang 790-784, Korea. ${ }^{4}$ IFW Dresden, Helmholtzstr. 20,01069 Dresden, Germany. ${ }^{5}$ Moscow State University, Moscow 119991, Russia. ${ }^{6}$ Institute of Experimental Mineralogy, Russian Academy of Sciences, Moscow 142432, Russia. ${ }^{7}$ Ural Federal University, Ekaterinburg 620002, Russia. ${ }^{8}$ Kazan Federal University, Kazan 420008, Russia. ${ }^{9}$ Department of Physics, Graduate School of Science, Tohoku University, Sendai $980-8578$, Japan. ${ }^{10}$ Department

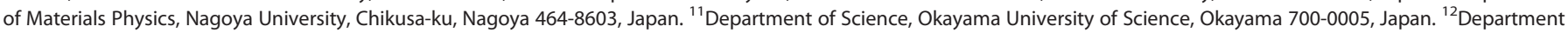
of Physics, Technische Universität Dresden, 01062 Dresden, Germany. *email: sbaek.fu@gmail.com
} 


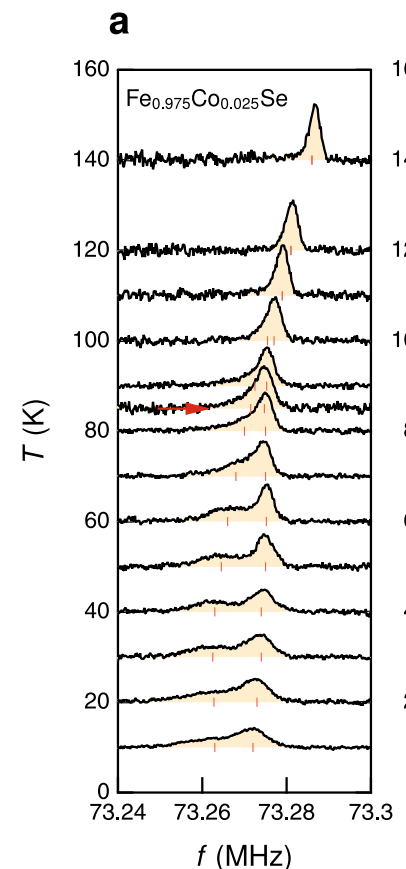

b

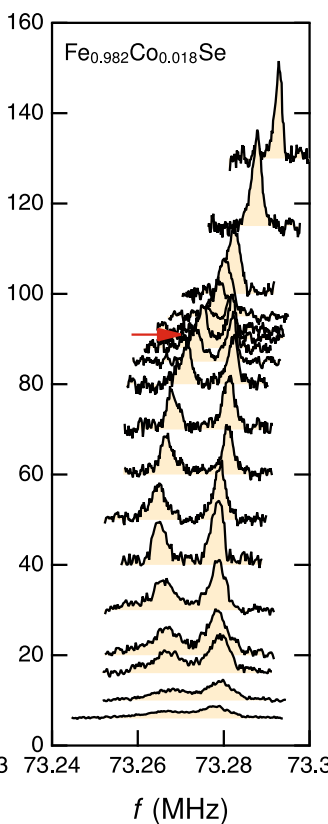

c

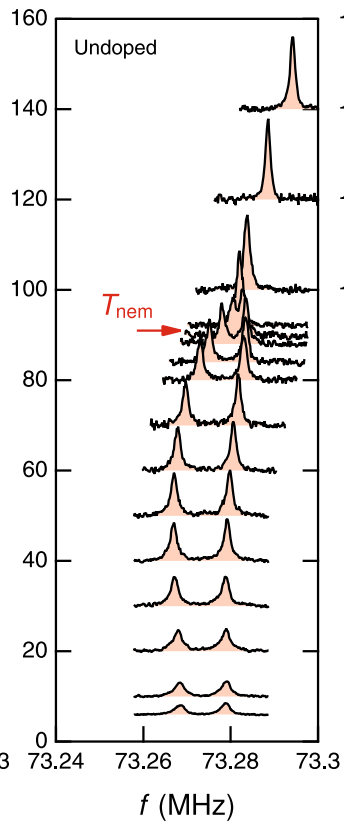

d

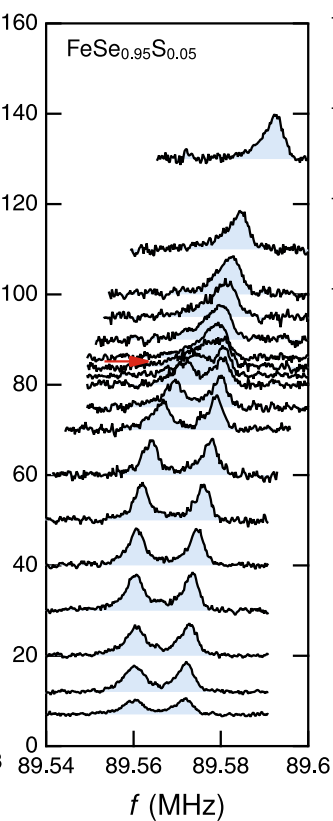

e

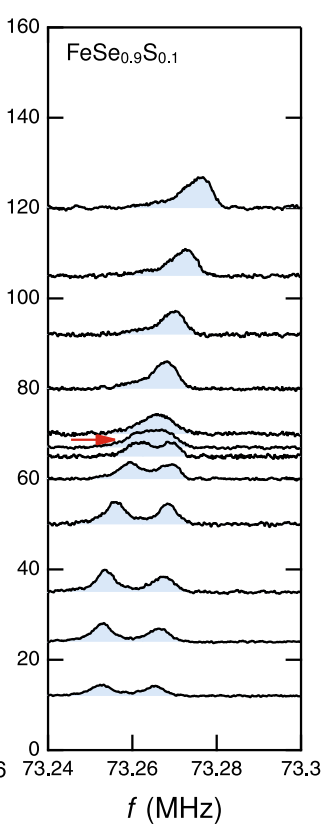

Fig. $1{ }^{77}$ Se NMR spectra in undoped and doped FeSe single crystals for $\boldsymbol{H} \| \boldsymbol{a} . \mathbf{a}, \mathbf{b}$ Temperature dependence of ${ }^{77} \mathrm{Se} \mathrm{spectrum} \mathrm{of} \mathrm{Fe}_{1-x} \mathrm{Co}_{x} \mathrm{Se}$. For $x=0.018(\mathbf{b})$, the ${ }^{77}$ Se spectrum shows a very similar behavior as the undoped one, except a moderate line broadening. For a slightly larger doping, $x=0.025$ (a), the ${ }^{77}$ Se line undergoes a considerable line broadening. While the splitting of the two ${ }^{77} \mathrm{Se}$ lines was clearly identified at low temperatures (vertical bars), the onset of the splitting is not well defined, being ascribed to local disorder. c Temperature dependence of ${ }^{77} \mathrm{Se}$ spectrum for undoped FeSe. d, e Temperature dependence of ${ }^{77} \mathrm{Se}$ spectrum of FeSe $\mathrm{F}_{1-y} \mathrm{~S}_{y}$ for $y=0.05$ and 0.1 , respectively. $T_{\text {nem }}$ is progressively suppressed with increasing $S$ doping.

changes for $x=0.018$. Upon further doping to slightly higher $x=$ 0.025 , the ${ }^{77}$ Se line becomes significantly broad, making it difficult to identify the onset of the line splitting. The much larger ${ }^{77}$ Se line broadening for Co doping than for $\mathrm{S}$ doping is well understood because Co has a strong influence on Fe moments as a nonmagnetic impurity. We notice, however, that the ${ }^{77} \mathrm{Se}$ line broadening is not simply proportional to the concentration of Co dopants, but rather it appears to increase drastically above $x \sim$ 0.025. In fact, for $x=0.036$, it is not possible to observe the line splitting anymore, because the linewidth is much larger than the nematic splitting (see Supplementary Fig. 1). On the other hand, the ${ }^{77} \mathrm{Se}$ linewidth is proportional to both $\mathrm{S}$ and Co dopants similarly, as long as Co doping is less than $2.5 \%$, as shown in Supplementary Fig. 2. This suggests that doped Co impurities beyond $\sim 2.5 \%$ of Fe sites cause a strong disorder effect on the correlation between Fe spins, indicating the existence of a critical doping level above which the magnetic correlation length becomes sufficiently long to induce a short-range exchange.

Although $T_{\text {nem }}$ cannot be accurately determined for $x=0.025$ due to the large line broadening in the nematic state, we clearly observed the line splitting below $80 \mathrm{~K}$, as shown in Fig. 1a. While this puts a lower limit of $T_{\text {nem }}$, the fitting analysis of ${ }^{77} \mathrm{Se}$ spectra in Fig. 1a also suggests that the line splitting seems to persist even up to $100 \mathrm{~K}$ (see vertical bars). (The detailed Knight shift data as a function of temperature and doping are shown in Supplementary Fig. 3). This suggests that Co impurities may induce a spatial distribution of $T_{\text {nem }}$ in the temperature range, $80 \leq T_{\text {nem }} \leq 100 \mathrm{~K}$. Regardless of the details, $T_{\text {nem }}$ is marginally suppressed by Co doping within the doping range investigated, as shown in Fig. 2a. Note that the Co-doping range investigated is very narrow, and thus we are unable to argue whether $T_{\text {nem }}$ remains a constant at higher Co doping. In any case, Fig. $2 \mathrm{a}$, b reveals that $T_{\text {nem }}$ and $T_{\mathrm{c}}$ are clearly decoupled.
Interestingly, the split ${ }^{77}$ Se lines below $80 \mathrm{~K}$ for $x=0.025$ are notably anisotropic, i.e., the peak for the lower frequency side is broader than that for the higher frequency. The origin of the anisotropic line shape is unclear, but we note that the similar anisotropic ${ }^{77} \mathrm{Se}$ line shape is also observed at $T<20 \mathrm{~K}$ for $x=$ 0.018 (see Fig. 1b). This implies that magnetic inhomogeneity, which otherwise appears at low temperatures, prevails at higher temperatures with higher Co doping.

Contrasting sharply with the weak dependence of nematicity on both S and Co dopants, our susceptibility measurements reveal that superconductivity is strongly dependent only on Co dopants. That is, $T_{\mathrm{c}}$ is rapidly suppressed by small Co doping, whereas it is robust with regard to $S$ doping, as shown in Fig. $2 a$, b, being consistent with previous studies. ${ }^{29,30}$ The very different behavior of $T_{\text {nem }}$ and $T_{c}$ with doping indicates that nematic and superconducting orders are not directly coupled (Coldea, A. I. et al., Evolution of the Fermi surface of the nematic superconductors $\mathrm{FeSe}_{1-x} \mathrm{~S}_{\mathrm{x}}$. arXiv:1611.07424, unpublished), ${ }^{31}$ raising a strong question as to whether nematicity and superconductivity are closely related. ${ }^{7,8,17,32}$

Having established the lack of a coupling of the nematic and superconducting transition temperatures, we now discuss the role of SFs for superconductivity. For probing low-energy SFs, we measured the spin-lattice relaxation rate, $T_{1}^{-1}$, as the quantity $\left(T_{1} T\right)^{-1}$ is a measure of SFs at very low energy

$$
\left(T_{1} T\right)^{-1}=\gamma_{n}^{2} \lim _{\omega \rightarrow 0} \sum_{\mathbf{k}} A^{2}(\mathbf{k}) \frac{X^{\prime \prime}(\mathbf{k}, \omega)}{\omega},
$$

where $\chi^{\prime \prime}(\mathbf{k}, \omega)$ is the imaginary part of the dynamic susceptibility at momentum $\mathbf{k}$ and frequency $\omega, \gamma_{n}$ is the nuclear gyromagnetic ratio, and $A(\mathbf{k})$ is the structure factor of the hyperfine interaction. Figure $2 \mathrm{C}$, $\mathrm{d}$ shows $\left(T_{1} T\right)^{-1}$ as a function of $\mathrm{Co}$ and $\mathrm{S}$ doping, respectively, at $H_{\| a}=9 \mathrm{~T}$. The data for the undoped FeSe crystal were taken from ref. ${ }^{26}$ With increasing Co-doping $x$ in 
a

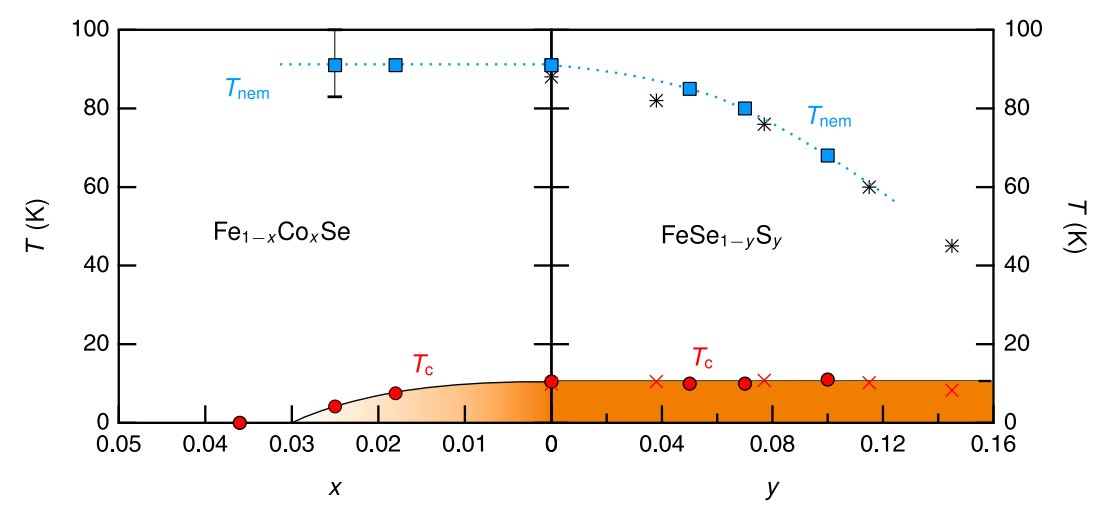

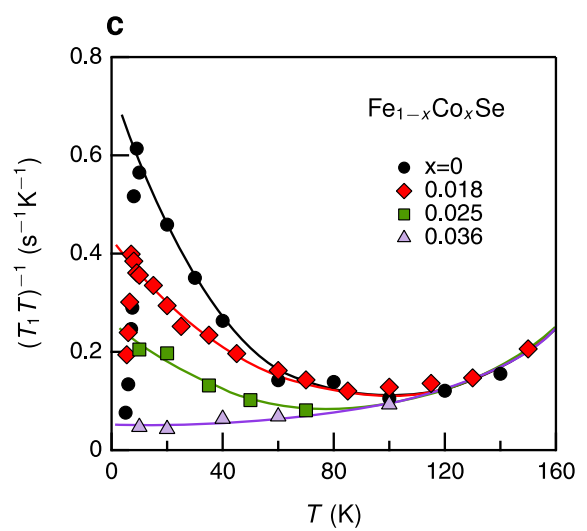

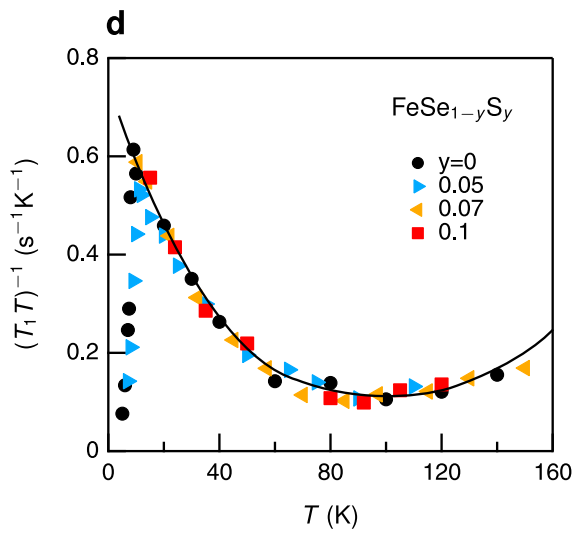

Fig. 2 Doping dependence of spin fluctuations for $\boldsymbol{H} \| \boldsymbol{a}$ in FeSe. $\mathbf{a}, \mathbf{b}$, The phase diagrams of $\mathrm{Fe}_{1-x} \mathrm{Co}_{x} \mathrm{Se}_{\text {and FeSe }} \mathrm{Fe}_{1-y} \mathrm{~S}_{y}$, respectively. For Co doping, $T_{\text {nem }}$ is hardly influenced, but $T_{\mathrm{c}}$ is strongly suppressed. In contrast, for $S$ doping, $T_{\text {nem }}$ is suppressed with increasing $y$, and $T_{\mathrm{c}}$ remains nearly the same. The asterisk $\left(^{*}\right)$ and cross $(x)$ symbols are the $T_{\text {nem }}$ and $T_{c}$ data, respectively, extracted from ref. ${ }^{17}$. c, The spin-lattice relaxation rate divided by temperature, $\left(T_{1} T\right)^{-1}$ which measures spin fluctuations, as a function of temperature and $\mathrm{Co}_{-} \mathrm{doping}_{x}$ in $\mathrm{Fe}_{1-x} \mathrm{Co}_{x} \mathrm{Se}_{\mathrm{T}} \mathrm{The}$ enhancement of $\left(T_{1} T\right)^{-1}$ at low temperatures is progressively suppressed with increasing $x$ (see Fig. 3). The solid lines are guides to the eyes. d, $\left(T_{1} T\right)^{-1}$ as a function of temperature and S-doping $y$ in $\mathrm{FeSe}_{1-y} \mathrm{~S}_{y}$. Spin fluctuations are unchanged with increasing S-doping $y$, being consistent with $T_{c}$ that is nearly independent of $y$.

$\mathrm{Fe}_{1-x} \mathrm{Co}_{x} \mathrm{Se},\left(T_{1} T\right)^{-1}$ or SFs above $T_{\mathrm{c}}$ is rapidly suppressed, which is exactly parallel with the suppression of $T_{\mathrm{c}}$, as shown in Fig. 2a. Note that for $x=0.035$, superconductivity is completely absent, and correspondingly SFs are not enhanced at all at low temperatures. On the other hand, $\left(T_{1} T\right)^{-1}$ above $T_{\mathrm{c}}$ is unchanged with increasing S-doping $y$ in $\mathrm{FeSe}_{1-y} \mathrm{~S}_{y}$ up to $y=0.1$, as precisely as $T_{c}$ does (see Fig. 2b). From the data presented in Fig. 2, therefore, one sees that $T_{\mathrm{c}}$ depends only on the strength of spin fluctuations, but not on $T_{\text {nem. }}$. (At larger $S$ doping near $y=0.2$, it was reported that both $\left(T_{1} T\right)^{-1}$ and $T_{\mathrm{c}}$ are strongly suppressed in such a way that the correlation between SFs and $T_{\mathrm{c}}$ persists, ${ }^{21}$ somewhat similar to the behavior in Co-doped samples).

For further quantitative information on how SFs are related to $T_{c}$ we adopt a spin fluctuation model in the Eliashberg formalism, ${ }^{33}$ or Millis-Monien-Pines (MMP) model. ${ }^{34}$ For this, we separate out the enhancement of $\left(T_{1} T\right)^{-1}$ that is solely associated with SFs from the data shown in Fig. 2c. Noting that $\left(T_{1} T\right)^{-1}$ for the non-superconducting sample $(x=0.035)$ approaches a constant, $\left(T_{1} T\right)_{0}^{-1} \equiv \Gamma_{0}$, without any enhancement at zero temperature, one may define the strength of SFs $\Gamma$ for $H_{\| a}$ from the $\left(T_{1} T\right)^{-1}(x)$ values just above $T_{\mathrm{c}}$ :

$\left.\Gamma(x) \equiv\left(T_{1} T\right)^{-1}(x)\right|_{T_{c}}-\Gamma_{0}$.

While the MMP model indicates that $\Gamma$ is proportional to the square of the correlation length, ${ }^{34} \xi^{2}(T)$, the estimation of the lowenergy part of the Eliashberg bosonic spectral function suggests that the coupling constant $\lambda$ is proportional to $\xi$, i.e., $\sqrt{\Gamma}$. As it was analyzed by Radtke et al. ${ }^{35}$ and Popovich et al., ${ }^{36}$ the direct use of the MMP spectrum gives overestimation of $T_{c}$ and the gap function due to a long tail at high energies of the bosonic spectral functions $\sim 1 / \omega$. To cure the problem, it was proposed to introduce a cutoff or calculate the bosonic self-energy at high energies. ${ }^{37}$ For simplicity, we use the approach of cutoff proposed in ref. ${ }^{36} \mathrm{~A}$ detailed procedure of the calculation is described in Supplementary Note 1.

The plot of $T_{c}$ vs. $\sqrt{\Gamma}$ is shown in Fig. 3. The solid curve is a theoretical calculation $\left(T_{c}\right.$ vs. $\lambda \propto \sqrt{\Gamma}$ ) based on the Eliashberg theory in which electron correlation effects are substantial. The good agreement of our theory with the experimental data evidences that the magnetic scenario for superconductivity in which Cooper pairing is mediated by spin fluctuations applies to $\mathrm{FeSe}$, and it is likely a universal superconducting mechanism among FeSCs.

Based on our NMR finding that $T_{\mathrm{c}}$ relies only on SFs, the seeming relevance of nematicity with superconductivity may be simply due to the closeness with magnetism, rather than to superconductivity itself. It should be noted that the strongly anisotropic gap structure ${ }^{13-16,22}$ observed in FeSe may be a natural consequence of the presence of nematicity within the superconducting state. It is because nematicity involves the splitting of $d_{x z}$ and $d_{y z}$ orbitals that should have an inevitable influence on the gap symmetry. However, $T_{c}$ itself is not necessarily affected by nematicity. ${ }^{38}$ Nevertheless, nematicity may be considered as an important barometer for 


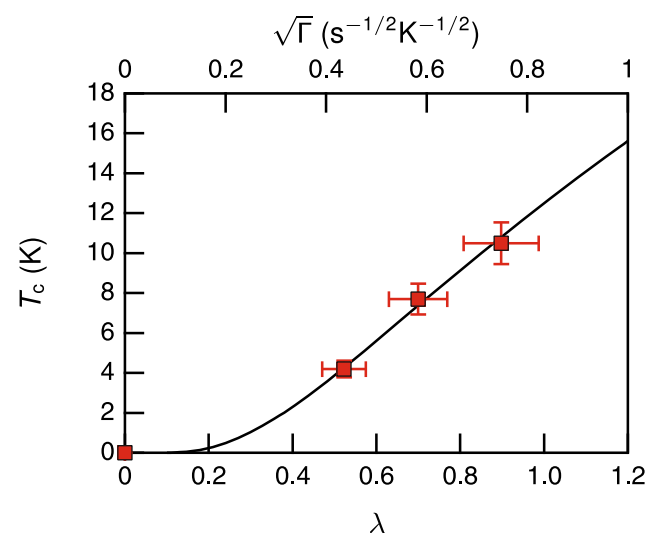

Fig. 3 Superconducting transition temperature $T_{\mathrm{c}}$ vs. $\sqrt{\Gamma}$, where $\Gamma$ is the strength of spin fluctuations just above $T_{c}$ for $H_{\| a}$ in Codoped FeSe single crystals. The solid line represents our theory of $T_{c}$ vs. the coupling constant $\lambda \propto \sqrt{\Gamma}$ (see text). The error bars represent the uncertainty in determining $T_{\mathrm{c}}$ and $\Gamma$.

superconductivity in FeSCs, as it is strongly coupled to magnetism, ${ }^{18}$ which in turn directly correlates with superconductivity.

\section{METHODS}

Crystal growth and characterization

The growth of $\mathrm{Fe}_{1-x} \mathrm{Co}_{x} \mathrm{Se}$ and $\mathrm{FeSe}_{1-y} \mathrm{~S}_{y}$ single crystals was performed by using the $\mathrm{KCl}-\mathrm{AlCl}_{3}$ flux technique in permanent $\mathrm{T}$ gradient in accordance with refs. ${ }^{39,40}$ All preliminary operations for the preparation of the reaction mixture were carried out in a dry box with a residual pressure of $\mathrm{O}_{2}$ and $\mathrm{H}_{2} \mathrm{O}$ not higher than $0.1 \mathrm{ppm}$. At the first stage, polycrystalline samples of the composition $\mathrm{Fe}_{1-x} \mathrm{Co}_{x} \mathrm{Se}$ and $\mathrm{FeSe}_{1-y} \mathrm{~S}_{y}$ were obtained. For this, $\mathrm{Fe}, \mathrm{Co}, \mathrm{S}$, and Se powders were carefully ground in a mortar in the appropriate ratio, and then annealed in evacuated quartz ampoules at $420^{\circ} \mathrm{C}$ for a few days. In the second stage, $0.5 \mathrm{~g}$ of the prepared sample was placed on the bottom of a thick-walled ampoule, and then the mixture of $\mathrm{AlCl}_{3}$ and $\mathrm{KCl}$ in a molar ratio of $\mathrm{AlCl}_{3}: \mathrm{KCl}=2: 1$ is added to the ampule, after that the ampule was evacuated and sealed. The sealed ampoule with polycrystalline sample of $\mathrm{Fe}-\mathrm{Co}-\mathrm{Se}-\mathrm{S}$ loads was placed in a horizontal two-zone furnace and heated for 5 weeks in such a way that the hot-zone temperature was set to $420^{\circ} \mathrm{C}$ and the cold-zone temperature was set to $370^{\circ} \mathrm{C}$. After 5 weeks, the furnace was turned off, and the ampoule was removed from the furnace. Next, the ampoule was cut, and the single crystals from the cold zone were separated from the flux by dissolving it in water. The single crystals obtained were thin square plates with metallic luster. The single crystals were grown with platelet-like morphology, and were characterized by SEM/EDX for compositional analysis.

\section{Nuclear magnetic resonance}

${ }^{77} \mathrm{Se}$ (nuclear spin $I=1 / 2$ ) NMR was carried out in undoped and doped FeSe single crystals at an external magnetic field, and in the range of temperature $4.2-160 \mathrm{~K}$. The samples were oriented using a goniometer for the accurate alignment along the external field. The ${ }^{77} \mathrm{Se}$ NMR spectra were acquired by a standard spin-echo technique with a typical $\pi / 2$ pulse length $2-3 \mu \mathrm{s}$. The nuclear spin-lattice relaxation rate $T_{1}^{-1}$ was obtained by fitting the recovery of the nuclear magnetization $M(t)$ after a saturating pulse to the following fitting function:

$1-M(t) / M(\infty)=A \exp \left(-t / T_{1}\right)$

where $A$ is a fitting parameter that is ideally unity.

Determination of $T_{\mathrm{C}}$ and $T_{\text {nem }}$

The superconducting transition temperature $T_{\mathrm{c}}$ was determined from magnetic susceptibility $(X)$ measurements by comparing field-cooled and zero-field-cooled data, while we obtained the nematic transition temperature $T_{\text {nem }}$ by measuring the temperature at which the ${ }^{77} \mathrm{Se}$ NMR line splits (see Fig. 1). Due to the weakness of the signal intensity, we were unable to determine $T_{\mathrm{c}}$ by $\left(T_{1} T\right)^{-1}$ measurements, except the undoped FeSe sample. This could give an error in extracting spin fluctuations just above $T_{\mathrm{c}}$ and $\Gamma$, which was reflected in an experimental error indicated in Fig. 3.

\section{DATA AVAILABILITY}

The data that support the findings of this study are available from the corresponding author (S-H.B.).

Received: 12 August 2019; Accepted: 7 January 2020; Published online: 30 January 2020

\section{REFERENCES}

1. Fradkin, E., Kivelson, S. A., Lawler, M. J., Eisenstein, J. P. \& Mackenzie, A. P. Nematic fermi fluids in condensed matter physics. Ann. Rev. Cond. Mat. Phys. 1, 153-178 (2010).

2. Fernandes, R. M., Chubukov, A. V. \& Schmalian, J. What drives nematic order in iron-based superconductors? Nature Phys. 10, 97-104 (2014).

3. Böhmer, A. E. \& Meingast, C. Electronic nematic susceptibility of iron-based superconductors. C. R. Physique 17, 90-112 (2016).

4. Si, Q., Yu, R. \& Abrahams, E. High-temperature superconductivity in iron pnictides and chalcogenides. Nat. Rev. Mater. 1, 16017 (2016).

5. Yamakawa, Y., Onari, S. \& Kontani, H. Nematicity and magnetism in FeSe and other families of Fe-based superconductors. Phys. Rev. X 6, 021032 (2016).

6. Chubukov, A. V., Khodas, M. \& Fernandes, R. M. Magnetism, superconductivity, and spontaneous orbital order in iron-based superconductors: which comes first and why? Phys. Rev. X 6, 041045 (2016).

7. Kuo, H.-H., Chu, J.-H., Palmstrom, J. C., Kivelson, S. A. \& Fisher, I. R. Ubiquitous signatures of nematic quantum criticality in optimally doped Fe-based superconductors. Science 352, 958-962 (2016).

8. Wang, C. G. et al. Electron mass enhancement near a nematic quantum critical point in $\mathrm{NaFe}_{1-x} \mathrm{Co}_{x}$ As. Phys. Rev. Lett. 121, 167004 (2018).

9. Wang, F. \& Lee, D.-H. The electron-pairing mechanism of iron-based superconductors. Science 332, 200-204 (2011).

10. Dai, P. Antiferromagnetic order and spin dynamics in iron-based superconductors. Rev. Mod. Phys. 87, 855-896 (2015).

11. She, J.-H., Lawler, M. J. \& Kim, E.-A. Quantum spin liquid intertwining nematic and superconducting order in FeSe. Phys. Rev. Lett. 121, 237002 (2018).

12. Böhmer, A. E. \& Kreisel, A. Nematicity, magnetism and superconductivity in FeSe. J. Phys.: Condens. Matter 30, 023001 (2018).

13. $\mathrm{Xu}, \mathrm{H}$. C. et al. Highly anisotropic and twofold symmetric superconducting gap in nematically ordered $\mathrm{FeSe}_{0.93} \mathrm{~S}_{0.07}$. Phys. Rev. Lett. 117, 157003 (2016).

14. Hashimoto, T. et al. Superconducting gap anisotropy sensitive to nematic domains in FeSe. Nat. Commun. 9, 282 (2018).

15. Liu, D. et al. Orbital origin of extremely anisotropic superconducting gap in nematic phase of FeSe superconductor. Phys. Rev. X 8, 031033 (2018).

16. Kushnirenko, Y. S. et al. Three-dimensional superconducting gap in FeSe from angle-resolved photoemission spectroscopy. Phys. Rev. B 97, 180501 (2018).

17. Hosoi, S. et al. Nematic quantum critical point without magnetism in $\mathrm{FeSe}_{1-x} \mathrm{~S}_{x}$ superconductors. Proc. Natl. Acad. Sci. USA 113, 8139-8143 (2016).

18. Matsuura, $\mathrm{K}$. et al. Maximizing $T_{c}$ by tuning nematicity and magnetism in $\mathrm{FeSe}_{1-x}$ $\mathrm{S}_{x}$ superconductors. Nat. Commun. 8, 1143 (2017).

19. Imai, T., Ahilan, K., Ning, F. L., McQueen, T. M. \& Cava, R. J. Why does undoped FeSe become a high- $T_{c}$ superconductor under pressure? Phys. Rev. Lett. 102, 177005 (2009).

20. Sun, J. P. et al. High- $T_{c}$ superconductivity in FeSe at high pressure: dominant hole carriers and enhanced spin fluctuations. Phys. Rev. Lett. 118, 147004 (2017).

21. Wiecki, P. et al. Persistent correlation between superconductivity and antiferromagnetic fluctuations near a nematic quantum critical point in $\mathrm{FeSe}_{1-x} \mathrm{~S}_{x}$. Phys. Rev. B 98, 020507 (2018).

22. Rhodes, L. C. et al. Scaling of the superconducting gap with orbital character in FeSe. Phys. Rev. B 98, 180503 (2018).

23. Wang, P. S. et al. Robust short-range-ordered nematicity in FeSe evidenced by high-pressure NMR. Phys. Rev. B 96, 094528 (2017).

24. Wiecki, P. et al. NMR evidence for static local nematicity and its cooperative interplay with low-energy magnetic fluctuations in FeSe under pressure. Phys. Rev. B 96, 180502 (2017).

25. Kuwayama, T. et al. ${ }^{77}$ Se-NMR study under pressure on $12 \%-S$ doped FeSe. J. Phys. Soc. Jpn. 88, 033703 (2019).

26. Baek, S.-H. et al. Orbital-driven nematicity in FeSe. Nature Mater. 14, 210-214 (2015). 
27. Baek, S.-H. et al. Nematicity and in-plane anisotropy of superconductivity in $\beta$ -FeSe detected by ${ }^{77}$ Se nuclear magnetic resonance. Phys. Rev. B 93, 180502 (2016).

28. Ok, J. M. et al. Nematicity and magnetism in LaFeAsO single crystals probed by ${ }^{75}$ As nuclear magnetic resonance. Phys. Rev. B. 97, 180405 (2018).

29. Urata, T. et al. Superconductivity pairing mechanism from cobalt impurity doping in FeSe: Spin $\left(s_{ \pm}\right)$or orbital $\left(s_{++}\right)$fluctuation. Phys. Rev. B 93, 014507 (2016).

30. Abdel-Hafiez, M. et al. Impurity scattering effects on the superconducting properties and the tetragonal-to-orthorhombic phase transition in FeSe. Phys. Rev. B 93, 224508 (2016).

31. Massat, $P$. et al. Collapse of critical nematic fluctuations in FeSe under pressure. Phys. Rev. Lett. 121, 077001 (2018).

32. Lederer, S., Schattner, Y., Berg, E. \& Kivelson, S. A. Enhancement of superconductivity near a nematic quantum critical point. Phys. Rev. Lett. 114, 097001 (2015).

33. Allen, P. B. \& Mitrović, B. Theory of superconducting $T_{c}$. Solid State Physics 37, 1-92 (1983).

34. Millis, A. J., Monien, H. \& Pines, D. Phenomenological model of nuclear relaxation in the normal state of $\mathrm{YBa}_{2} \mathrm{Cu}_{3} \mathrm{O}_{7}$. Phys. Rev. B 42, 167-178 (1990).

35. Radtke, R. J., Ullah, S., Levin, K. \& Norman, M. R. Constraints on superconducting transition temperatures in the cuprates: antiferromagnetic spin fluctuations. Phys. Rev. B 46, 11975-11985 (1992).

36. Popovich, $\mathrm{P}$. et al. Specific heat measurements of $\mathrm{Ba}_{0.68} \mathrm{~K}_{0.32} \mathrm{Fe}_{2} \mathrm{As}_{2}$ single crystals: evidence for a multiband strong-coupling superconducting state. Phys. Rev. Lett. 105, 027003 (2010)

37. Abanov, A., Chubukov, A. V. \& Schmalian, J. Quantum-critical theory of the spinfermion model and its application to cuprates: normal state analysis. Adv. Phys. 52, 119-218 (2003)

38. Kang, J., Fernandes, R. M. \& Chubukov, A. Superconductivity in FeSe: the role of nematic order. Phys. Rev. Lett. 120, 267001 (2018).

39. Chareev, D. et al. Single crystal growth and characterization of tetragonal $\mathrm{FeSe}_{1-x}$ superconductors. CrystEngComm 15, 1989-1993 (2013).

40. Chareev, D. et al. Single crystal growth, transport and scanning tunneling microscopy and spectroscopy of $\mathrm{FeSe}_{1-x} \mathrm{~S}_{x}$. CrystEngComm 20, 2449-2454 (2018).

\section{ACKNOWLEDGEMENTS}

We acknowledge A. Chubukov for useful discussions. S.H.B. has been supported by the Deutsche Forschungsgemeinschaft (Germany) via DFG Research Grants BA 4927/ 2-1 and by the National Research Foundation of Korea (NRF-2019R1F1A1057463). D.V. E., B.B., I.M., and S.A. were supported by RSF-DFG project (no. 19-43-04129, BU887/251, EF86/7-1). D.V.E. and I.M. were also supported by VW foundation in the frame of the VW Trilateral Initiative. S.A. acknowledges financial support from Deutsche Forschungsgemeinschaft (DFG) via Grant No. DFG AS 523/4-1. The work at POSTECH was supported by Institute for Basic Science (no. IBS-R014-D1) and also by the National Research Foundation (NRF) of Korea through the SRC (no. 2018R1A5A6075964) and the Max Planck-POSTECH Center for Complex Phase
Materials in Korea (MPK) (no. 2016K1A4A4A01922028). The work of DACh was supported by the program 211 of the Russian Federation Government, agreement No. 02.A03.21.0006, by the Russian Government Program of Competitive Growth of Kazan Federal University.

\section{AUTHOR CONTRIBUTIONS}

S-H.B. and B.B. have proposed and initiated the project. J.M.O., J.S.K., S.A., I.M., D.C., T.U., K.T. and Y.T. have grown single crystals and characterized transport and superconducting properties. S-H.B. and J.M.O. performed NMR measurements and analyzed data; S-H.B., D.V.E. and B.B. participated in writing of the paper. All authors discussed the results and commented on the paper.

\section{COMPETING INTERESTS}

The authors declare no competing interests.

\section{ADDITIONAL INFORMATION}

Supplementary information is available for this paper at https://doi.org/10.1038/ s41535-020-0211-y.

Correspondence and requests for materials should be addressed to S.-H.B.

Reprints and permission information is available at http://www.nature.com/ reprints

Publisher's note Springer Nature remains neutral with regard to jurisdictional claims in published maps and institutional affiliations.

Attribution 4.0 International License, which permits use, sharing, adaptation, distribution and reproduction in any medium or format, as long as you give appropriate credit to the original author(s) and the source, provide a link to the Creative Commons license, and indicate if changes were made. The images or other third party material in this article are included in the article's Creative Commons license, unless indicated otherwise in a credit line to the material. If material is not included in the article's Creative Commons license and your intended use is not permitted by statutory regulation or exceeds the permitted use, you will need to obtain permission directly from the copyright holder. To view a copy of this license, visit http://creativecommons. org/licenses/by/4.0/.

(c) The Author(s) 2020 